\title{
The immediate effectiveness of mobile game-based instruction with an extracorporeal biofeedback device for an exercise program to improve pelvic floor muscle contraction in healthy subjects
}

\author{
Hyo Jeong Kang ${ }^{a(1)}$, Mi-Hwa Kim ${ }^{a}$, Ji Hye Hwang ${ }^{b(1)}$, Wan-Hee Lee ${ }^{c(1)}$ \\ ${ }^{a}$ Department of Physical Therapy, Cheongam College, Suncheon, Republic of Korea \\ ${ }^{b}$ Department of Physical and Rehabilitation Medicine, Samsung Medical Center, Sungkyunkwan University School of Medicine, Seoul, \\ Republic of Korea \\ 'Department of Physical Therapy, College of Health Science and Social Welfare, Sahmyook University, Seoul, Republic of Korea
}

Objective: Using biofeedback in instructing pelvic floor muscle (PFM) activation is a great method to provide information on muscle contraction. This study aimed to determine the immediate effectiveness of a mobile game-based instruction with an extracorporeal biofeedback device (EBD) to improve PFM contraction in healthy subjects.

Design: Cross-sectional study.

Methods: Sixteen healthy subjects (4 men and 12 nulliparous women; age, $31 \pm 5$ years) were enrolled. The subjects were randomly categorized into two groups, those who were to receive instructions on how to contract the PFM by using the EBD (n=7) and those who were to undergo a transabdominal ultrasonography (TAUS; $n=9$ ) with biofeedback. PFM function was measured as the displacement (mm) of PFM elevation by using the TAUS before and after the instructions in each group.

Results: The EBD and TAUS groups showed a significant increase in the displacement of PFM elevation after the instructions from $5.93 \pm 4.03 \mathrm{~mm}$ to $7.62 \pm 3.77 \mathrm{~mm}$ and from $5.27 \pm 3.39 \mathrm{~mm}$ to $7.47 \pm 2.79 \mathrm{~mm}$, respectively $(p<0.05)$. No significant differences were found between the two groups.

Conclusions: The results of this study indicated that instructions for PFM contraction using the EBD and TAUS showed an immediate effect; however, no significant difference in effectiveness was observed between the two instruction methods. Therefore, not only can the TAUS method be used but the EBD method can also be used as a PFM instruction method for noninvasive procedures. However, further studies are needed to demonstrate the effectiveness of training and exercise on larger sample sizes that includes patient populations with PFM dysfunction.

Key Words: Extracorporeal biofeedback, Game-based education, Pelvic floor muscle, Transabdominal ultrasonography

\section{Introduction}

The pelvic floor muscle (PFM) are a collection of muscles and ligaments that form the pelvic hammock below the pelvis, supporting the bladder, rectum and uterus (in woman). The function of the PFM are to maintain continence as part of the urinary and anal sphincters, in addition to contract in cooperation with the deep abdominal muscles and diaphragm to assist in maintaining intra-abdominal pressure. Moreover, it performs various functions such as maintaining pelvic stability, posture control, and providing breathing assistance. Malfunctions due to PFM weakness or impairment disrupt the mechanism that counteracts the increase in abdominal pressure and can lead to incontinence, pelvic or-

Received: 31 August, 2020 Revised: 17 September, 2020 Accepted: 17 September, 2020

Corresponding author: Wan-Hee Lee (ORCID https://orcid.org/0000-0001-8030-4853)

Department of Physical Therapy, College of Health Science and Social Welfare, Sahmyook University, 815 Hwarang-ro, Nowon-gu, Seoul 01795, Republic of Korea Tel: 82-2-3399-1633 Fax: 82-2-3399-1639 E-mail: whlee@syu.ac.kr

(c) This is an Open-Access article distributed under the terms of the Creative Commons Attribution Non-Commercial License (http://creativecommons.org/licenses/ by-nc/4.0) which permits unrestricted non-commercial use, distribution, and reproduction in any medium, provided the original work is properly cited.

Copyright @ 2020 Korean Academy of Physical Therapy Rehabilitation Science 
gan prolapse, and pelvic and lower back pains [1].

Independent and repetitive contraction of the PFM for the prevention and treatment of pelvic floor dysfunction was reported in 1948 by Arnold Kegel, who argued that the recovery rate for various type of incontinence was $84 \%$ [2]. However, the isolated voluntary PFM contraction is difficult to maintain because the muscle is not located within a visible range and is attached to immovable joints.

Voluntary contraction of the PFM is performed accurately at less than $50 \%$ of the time when training and exercises are performed with verbally guided instructions [3]. Caagbay et al. [4] reported that verbal explanations using leaflets on how to contract the PFM were ineffective in subjects with low literacy, limited resources, and or those living in poor settings. The biofeedback method is effective and useful for promoting awareness and control of PFM contraction. It provides information on muscle contraction in real time, and for this reason, it is used as patient education in regards to voluntary PFM contraction [5]. The visual biofeedback research by Engel et al. [6] in 1974 for effective education and training of PFM contraction is still being studied for its method and effectiveness 40 years later. The main approaches to visual feedback include the use of a perineometer, manometry, electromyography, and real-time ultrasonography [7]. Most methods require insertion of a probe or an electrode in the vagina or rectum to monitor muscle activity and squeeze pressures exerted by the PFM. This may cause patient reproach or rejection, and may interfere with receiving instruction and training, and it is limited in application when surgery or treatment is performed on or around the insertion site [8].

Therefore, noninvasive visual feedback methods such as transperineal ultrasonography (TPUS) and transabdominal ultrasonography (TAUS) have been experimented according to a recent study, because they can inspect the PFM through contact with the body surface without inserting a probe [9]. Instead of the TPUS, in which a probe is directly in contact with the perineal area, the TAUS is mainly used to confirm the degree of PFM contraction by imaging the base of the bladder by contact of the probe with the lower abdomen. However, the TAUS is also difficult because the patient is instructed to have a full bladder by drinking water an hour before the monitoring.

However, PFM contraction education, training, and rehabilitation using computer games [10], virtual reality games [11], and game therapy [12] have recently been proposed and gained increasing interest as noninvasive methods. These methods are performed using visual feedback, but most programs only train the PFM indirectly by contracting the muscles of the torso that are related to the PFM. Lee et al. [8] published a study, which showed that symptoms and muscle contraction were improved by game-based training using an extracorporeal biofeedback device (EBD) with a pressure sensor in patients with urinary incontinence. However, the limitation of the study was that the quantitative contractile force of the PFM was difficult to confirm using the Oxford scale, which evaluates the contractile force of the PFM on a 5-point scale by inserting the evaluator's index finger into the vagina. Furthermore, it is a computer-based game therapy that cannot be used in a limited space and is expensive. Game therapy using a mobile phone is useful as a home training tool because it is portable and inexpensive. However, only a few studies have been conducted on the use of mobile phone game therapy to educate and train the PFM contraction so far.

Therefore, this study aimed to confirm the possibility of education and training for PFM contraction using a mobile game-based EBD with a pressure sensor that can visualize PFM contractions in healthy adults. This study was conducted to compare and analyze the immediate effectiveness of the therapy by performing functional tests of the PFM using TAUS to provide education.

\section{Methods}

\section{Participants}

The study was conducted with 16 healthy adults who met the selection criteria from among 22 volunteers who participated in the experiment. We used the $G^{*}$ Power version 3.1.9.2 (Heinrich-Heine-Universität, Düsseldorf, Germany) to calculate the sample size of study subjects. According to the results of a previous study, the sample size calculated using a test power $(\rho)$ of 0.90 and the significance level of 0.05 was 22 [8]. An IRB-approved poster that explained the purpose and method of this study was used to recruit the subjects. The selection criteria were subjects aged 18 to 40 years, and those with normal weight as indicated by a body mass index (BMI) between 18.5 and $25.0 \mathrm{~kg} / \mathrm{m}^{2}$. The exclusion criteria were subjects with urinary and fecal incontinence who have symptoms of constipation for the past 3 months or more, those who had undergone surgical surgery within the last year; those who have been diagnosed as having neurological, mental, and cardiovascular diseases, and respiratory musculoskeletal disorders; and pregnant and 
postpartum women. All the subjects provided voluntary consent after hearing the explanation of the purpose and method of the study. The study was approved by the Institutional Review Board of the Institutional Bioethics Committee of Sahmyook University (IRB No. 2-1040781AB-N-01-2016108HR).

\section{Outcome assessments}

The subjects were assessed with the PFM function test using ultrasonography (3D TRUS Accuvix V10; Samsung Medison Co., Ltd., Seongnam, Korea) by TAUS at two time points: immediately before and after education of the PFM contraction. The distance of the movement of the bladder base was calculated at rest and during the PFM contraction for the PFM function test. The location of the base of the bladder was measured using a $3.5 \mathrm{~Hz}$ curved linear array ultrasonographic transducer probe. To fill the bladder, it was emptied 1 hour before the test and filled by drinking $500 \mathrm{ml}$ of water. The measurement was performed in a crook-lying supine position which is to bend the hip and knee joints by $60^{\circ}$ in the supine position. The examiner placed the probe on the abdomen $5 \mathrm{~cm}$ above the pubic symphysis region of subjects, and tilted it at $15^{\circ}-30^{\circ}$. The arrow indicated the lowest point of the base of the bladder at rest and the image was captured. Then, the examiner marked with an arrow again to indicate the bladder base at the highest maximum displacement when the participant performed maximum PFM contraction. The probe was not moved during the testing procedure (Figure 1) [9].

\section{Research procedure}

The subjects were divided randomly into two groups according to the PFM contraction method used as follows: the mobile game-based EBD group and the TAUS group. Before the experiment, all subjects received full explanations and understood the information of the ex-

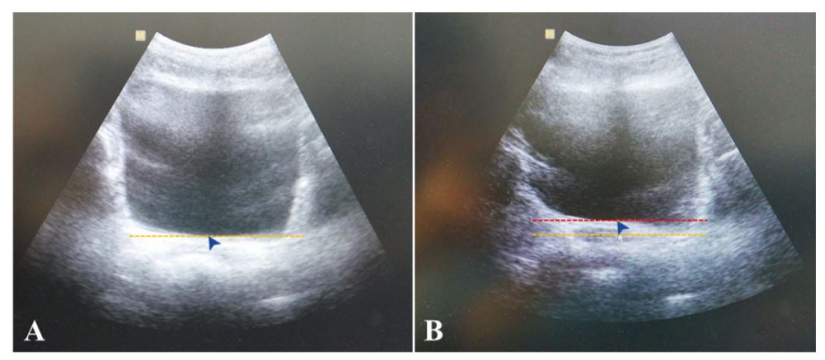

Figure 1. Pelvic floor muscle (PFM) function test using the transabdominal ultrasound images. (A) Rest. (B) PFM contraction. perimental procedure and how to contract the PFM.

To conduct the PFM contraction visual feedback education using a mobile game-based EBD (Any kegel; Furun Healthcare, Wonju, Korea), an application was installed in a mobile phone (Galaxy S7; Samsung, Suwon, Korea). At first, the subjects wore thin clothes to be able to feel the force more effectively and then sat on the center of the pressure sensor of the EBD, which is located on a chair with the hip and knee joint at approximately $90^{\circ}$ of flexion. A pressure sensor was placed between the buttocks, positioned between the anus and the urethra, to contact the PFM. Calibration was performed while the subject sat comfortably on the pressure sensor without contracting the PFM. After the full explanation of the game method was provided to the subject, the game was played for 8 minutes ( 2 minutes/set, total 3 sets, each set with a 30 second rest) while looking at the mobile phone located $1 \mathrm{~m}$ in front of the subject and the subject was educated on how to contract and relax the PFM through visual feedback. The method involved touching a coin while drawing a parabolic line and the balloon floating on the screen continuously that moved from left to right, rising up according to the degree of PFM contraction, and descending when the PFM relaxed (Figure 2).

Visual feedback education using the TAUS was conducted under the same conditions as the PFM function test. At this time, the subject could see the ultrasonography image through the monitor. The subject received information to assist in understanding the ultrasonography image, which was the basal surface of the risen bladder when the PFM contracted, and at the descended position with the PFM relaxed. Thus, the subject was then instructed to relax for 5 seconds after a maximum contraction of 10 seconds, and visual feed-

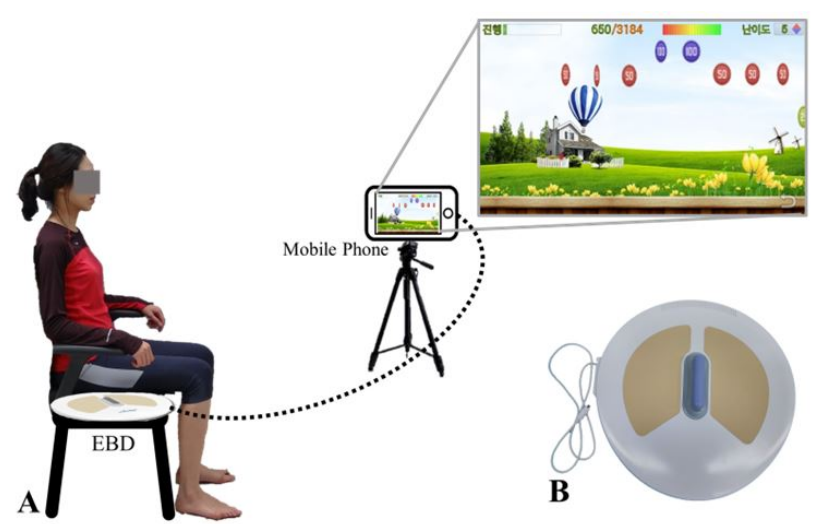

Figure 2. Education using a mobile game-based extracorporeal biofeedback device (EBD). (A) Posture during the experiment. (B) Any kegel (Furun Healthcare). 
back training was conducted for 8 minutes (10 times/set, total 3 sets) [9].

\section{Data processing}

In this study, statistical analysis was performed using IBM SPSS Statistics for Windows, Version 22.0 (IBM Co., Armonk, NY, USA), and the general characteristics of the subjects were analyzed using descriptive statistics, Chi square test, and Mann-Whitney U-test. The Wilcoxon signed-rank test was performed to confirm the pre- and post-group differences, and the Mann-Whitney U-test was performed to confirm the difference in PFM function between the two groups. All statistical significance levels $(\alpha)$ were set to $p<0.05$.

\section{Results}

No significant differences were found between the two groups in terms of age, height, weight, and BMI (Table 1). The displacement of the PFM elevation significantly increased after the instructions from $5.93 \pm 4.03 \mathrm{~mm}$ to $7.62 \pm 3.77 \mathrm{~mm}(p<0.05)$ in the EBD education group, with a mean increase rate of $38 \%$ (range, $4 \%-80 \%$ ), and from $5.27 \pm 3.39 \mathrm{~mm}$ to $7.47 \pm 2.79 \mathrm{~mm}(p<0.05)$, with an increase rate of $75 \%$ (range, $4 \%-180 \%$ ) in the TAUS education group. No significant differences in PFM elevation dis- placement was found between the two teaching methods after the instructions (Table 2).

\section{Discussion}

Voluntary contraction of the PFM controls urine, gas, and bowel movements by tightening the vagina, urethra, and rectum, respectively. However, performing accurate voluntary contractions is difficult for patients with conditions such as urinary and fecal incontinence, and for healthy people. In some cases, symptoms may not improve or may actually worsen by increasing the abdominal pressure through incorrect training methods for PFM contraction. A visual feedback training method is used to recognize and educate on PFM contractions. It is a useful method for visually confirming muscle contraction using ultrasonography techniques such as TAUS and TPUS, which do not involve directly inserting an ultrasonography probe in the vagina or rectum. However, the application of this method is not easy clinically because it requires a long preparation time and much effort before implementation. Therefore, nowadays, a game-based intervention method with an EBD is being developed for patients to induce voluntary contraction of the PFM, with the use of technological developments such as computers, mobile phones, and internet of things.

The present study compared the immediate effectiveness

Table 1. General characteristics of the subjects

\begin{tabular}{lccc}
\hline \multicolumn{1}{c}{ Variable } & TAUS group $(\mathrm{n}=9)$ & EBD group $(\mathrm{n}=7)$ & $p$-value \\
\hline Gender (man/woman) & $1 / 8$ & $3 / 4$ & 0.262 \\
Age $(\mathrm{y})$ & $31.44(6.60)$ & $30.43(3.69)$ & 0.487 \\
Height $(\mathrm{cm})$ & $166.67(6.69)$ & $166.71(7.80)$ & 0.312 \\
Weight $(\mathrm{kg})$ & $157.67(9.04)$ & $58.71(7.83)$ & 0.958 \\
BMI $\left(\mathrm{kg} / \mathrm{m}^{2}\right)$ & $20.68(2.28)$ & $221.10(1.98)$ & 0.368 \\
\hline
\end{tabular}

Values are presented as number only or mean (SD).

TAUS: transabdominal ultrasonography, EBD: extracorporeal biofeedback device, BMI: body mass index.

Table 2. Comparison of pre- and post- instruction of PFM functions in terms of displacement (in millimeters) of PFM elevation between the two groups

\begin{tabular}{lccc}
\hline \multicolumn{1}{c}{ PFM functions } & TAUS group $(\mathrm{n}=9)$ & EBD group $(\mathrm{n}=7)$ & $p$-value \\
\hline Pre-instruction & $5.27(3.39)$ & $5.93(4.03)$ & \\
Post-instruction & $7.47(2.79)$ & $7.62(3.77)$ & 0.529 \\
Difference (post-pre) & $2.20(1.67)$ & $1.68(1.26)$ & 0.028 \\
$p$-value & 0.008 & & \\
\hline
\end{tabular}

Values are presented as mean (SD).

PFM: pelvic floor muscle, TAUS: transabdominal ultrasonography, EBD: extracorporeal biofeedback device. 
of using the TAUS and mobile game-based education using the EBD to confirm the possibility of education and training for voluntary PFM contraction in healthy adults. This study showed that the PFM contractile force in the education group using the TAUS was significantly increased from $5.27 \pm 3.39 \mathrm{~mm}$ to $7.47 \pm 2.79 \mathrm{~mm}$, with a mean rate of change of $75 \%$. Dietz et al. [12] reported that among women who were able to contract their PFM voluntarily, 57\% could not correctly contract the PFM after training for 5 minutes using the TPUS [12]. This difference is thought to be because of the fact that this study was conducted in healthy adults without symptoms rather than in a disease group whose voluntary PFM contractility was decreased before education.

The EBD education group showed a significant increase in displacement of the PFM elevation from $5.93 \pm 4.03 \mathrm{~mm}$ to $7.62 \pm 3.77 \mathrm{~mm}(p<0.05)$, with a mean rate of change of $38 \%$. Silva et al. [13] applied game therapy for contraction training of the PFM and abdominal muscles in young women and reported that coactivation of the PFM using electromyography increased significantly from 127.27 to 147.84 .

According to the results of this study, PEM contraction education using the EBD and TAUS showed immediate effectiveness, which did not significantly differ between the two education methods. Therefore, these results demonstrate the possibility of using not only the method using the TAUS but also that method using the EBD as a non-insertion method of PFM contraction education.

Low effectiveness of PFM contraction education and training conducted through oral explanations has been reported [3], Caagbay et al. [4] did not use leaflet verbal explanations in subjects with low literacy level. For effective education and training of the PFM, the visual feedback method introduced by Engel et al. [6] in 1974 has been used continuously on the subjects to investigate its procedures and effects even after 40 years.

In this study, an EBD based on mobile games was used to confirm the effectiveness of PFM contraction education. The EBD and TAUS methods did not show statistically significant difference, showing the potential usefulness of the EBD method for PFM contraction. However, the EBD method was shown to be less effective than the TAUS method using visual feedback for education. The limitations of this study include the small sample size, and the fact that it was conducted in healthy adults. Also, PFM contraction education was conducted with 8 minutes of training time in this study. A previous study showed an effect with 5 minutes of visual feedback training [12], but there are still a lack of re- search on effective training time. Therefore, future studies are needed to compare effective training and education protocols. In addition, further studies are needed to verify the suitable methods of education in subjects with urinary and fecal incontinence, genitourinary cancer treatment, and surgical diseases, which cause functional abnormalities of the PFM.

In conclusion, in this study, mobile game-based instruction with an EBD was demonstrated to have potential usefulness as an education and training method for voluntary PFM contraction in healthy subjects.

\section{Conflict of Interest}

The authors declared no potential conflicts of interest with respect to the authorship and/or publication of this article.

\section{References}

1. Bø K, Berghmans B, Mørkved S, Van Kampen M. Evidencebased physical therapy for the pelvic floor: bridging science and clinical practice. 2nd ed. Philadelphia (PA): Churchill Livingstone; 2014.

2. Kegel AH. Progressive resistance exercise in the functional restoration of the perineal muscles. Am J Obstet Gynecol 1948;56: 238-48.

3. Bump RC, Hurt WG, Fantl JA, Wyman JF. Assessment of Kegel pelvic muscle exercise performance after brief verbal instruction. Am J Obstet Gynecol 1991;165:322-7; discussion 327-9.

4. Caagbay DM, Black K, Dangal G, Raynes-Greenow C. Can a leaflet with brief verbal instruction teach Nepali women how to correctly contract their pelvic floor muscles? J Nepal Health Res Counc 2017; 15:105-9.

5. Ibrahim IK, Hameed MM, Taher EM, Shaheen EM, Elsawy MS. Efficacy of biofeedback-assisted pelvic floor muscle training in females with pelvic floor dysfunction. Alexandria J Med 2015; 51:137-42.

6. Engel BT, Nikoomanesh P, Schuster MM. Operant conditioning of rectosphincteric responses in the treatment of fecal incontinence. N Engl J Med 1974;290:646-9.

7. Scott KM. Pelvic floor rehabilitation in the treatment of fecal incontinence. Clin Colon Rectal Surg 2014;27:99-105.

8. Lee HN, Lee SY, Lee YS, Han JY, Choo MS, Lee KS. Pelvic floor muscle training using an extracorporeal biofeedback device for female stress urinary incontinence. Int Urogynecol J 2013;24: 831-8.

9. Ariail A, Sears T, Hampton E. Use of transabdominal ultrasound imaging in retraining the pelvic-floor muscles of a woman postpartum. Phys Ther 2008;88:1208-17.

10. McKenna PH, Herndon CD, Connery S, Ferrer FA. Pelvic floor muscle retraining for pediatric voiding dysfunction using interactive computer games. J Urol 1999;162(3 Pt 2):1056-62; dis- 
cussion 1062-3.

11. Botelho S, Martinho NM, Silva VR, Marques J, Carvalho LC, Riccetto C. Virtual reality: a proposal for pelvic floor muscle training. Int Urogynecol J 2015;26:1709-12.

12. Dietz HP, Wilson PD, Clarke B. The use of perineal ultrasound to quantify levator activity and teach pelvic floor muscle exercises.
Int Urogynecol J Pelvic Floor Dysfunct 2001;12:166-8; discussion 168-9.

13. Silva VR, Riccetto CL, Martinho NM, Marques J, Carvalho LC, Botelho S. Training through gametherapy promotes coactivation of the pelvic floor and abdominal muscles in young women, nulliparous and continents. Int Braz J Urol 2016;42:779-86. 